16 | 2021

Simone et André Schwarz-Bart : nouvelles approches de l'oeuvre

\title{
« La femme Solitude de Guadeloupe »
}

Ce que nous dit l'avant-texte de La Mulâtresse Solitude

\section{Catherine Rovera}

\section{(2) OpenEdition}

\section{Journals}

Édition électronique

URL : https://journals.openedition.org/coma/6673

DOI : $10.4000 /$ coma. 6673

ISSN : 2275-1742

Éditeur

Institut des textes \& manuscrits modernes (ITEM)

Référence électronique

Catherine Rovera, « «La femme Solitude de Guadeloupe » », Continents manuscrits [En ligne], 16 | 2021, mis en ligne le 02 mars 2021, consulté le 18 août 2022. URL : http://journals.openedition.org/coma/ 6673 ; DOI : https://doi.org/10.4000/coma.6673

Ce document a été généré automatiquement le 18 août 2022.

\section{(2) $(\mathcal{Q} \Theta \Theta$}

Creative Commons - Attribution - Pas d'Utilisation Commerciale - Pas de Modification 4.0 International - CC BY-NC-ND 4.0

https://creativecommons.org/licenses/by-nc-nd/4.0/ 


\title{
« La femme Solitude de Guadeloupe »
}

Ce que nous dit l'avant-texte de La Mulâtresse Solitude

\author{
Catherine Rovera
}

\begin{abstract}
Ce petit livre est à la fois totalement réaliste et totalement fictif. Une certaine mulâtresse a existé dont on ne sait pas grand-chose, sinon sa participation à la lutte contre l'esclavage et son supplice. Tout le reste est fiction, bien qu'étant extrêmement fidèle, je l'espère, à la vérité historique, anthropologique \& humaine ${ }^{1}$.
\end{abstract}

\section{Béance(s) de l'avant-texte}

Lorsque le roman La Mulâtresse Solitude $e^{2}$ paraît en 1972, il succède à Un plat de porc aux bananes vertes et appartient alors au même cycle romanesque. Mais étant signé d'André Schwarz-Bart seul, il introduit une pause dans l'écriture à quatre mains et constitue par là même « une rupture dans le déroulement prévu du cycle ». Dans son avant-propos pour L'Ancêtre en Solitude, c'est en ces termes en effet que Simone Schwarz-Bart analyse, avec le recul, le besoin impérieux qu'elle a eu de laisser à André le soin d'écrire et de publier en son nom seul le second volume du cycle antillais :

À l'époque j'écrivais Pluie et vent sur Télumée Miracle. J'étais en mal d'Ancêtre. Je ressentais une béance et notre histoire n'en finissait pas de me manquer. J'avais besoin de cette histoire secrète des vérités originelles, et j'étais moi-même incapable de l'écrire. Et c'est sur ce besoin absolu que la décision de publier $L a$ Mulâtresse Solitude a été arrêtée. Le livre paraît en 1972, et c'est une rupture dans le déroulement prévu du cycle ${ }^{3}$.

2 Plus encore que l'oubli ou le silence, la «béance » dont il est question ici serait cette faille primordiale, ce néant sur et par lequel la nation antillaise s'est construite, et que le roman interroge loin de tout exotisme. Chercher à les combler serait illusoire, tant l'amnésie fait partie intégrante de l'inconscient collectif guadeloupéen. Parce qu'il 
exhume une figure phare de la révolte guadeloupéenne jusqu'alors inconnue de son propre peuple tout en réussissant le pari de la faire passer à la postérité, le roman répond bien à la nécessité de transmettre à la communauté antillaise - et humaine des bribes de son histoire fondatrice. C'est tout à la fois cette "rupture " et cette "béance» que je souhaiterais analyser ici à la lumière des lettres, manuscrits et brouillons du roman. Car revenir à cette "histoire secrète des vérités originelles " implique de revenir aux sources mêmes de l'œuvre, autrement dit à l'avant-texte.

Fig. 1 et $2^{4}$ : Page de titre du roman, t. II du cycle (à gauche). Dessin autographe (à droite).
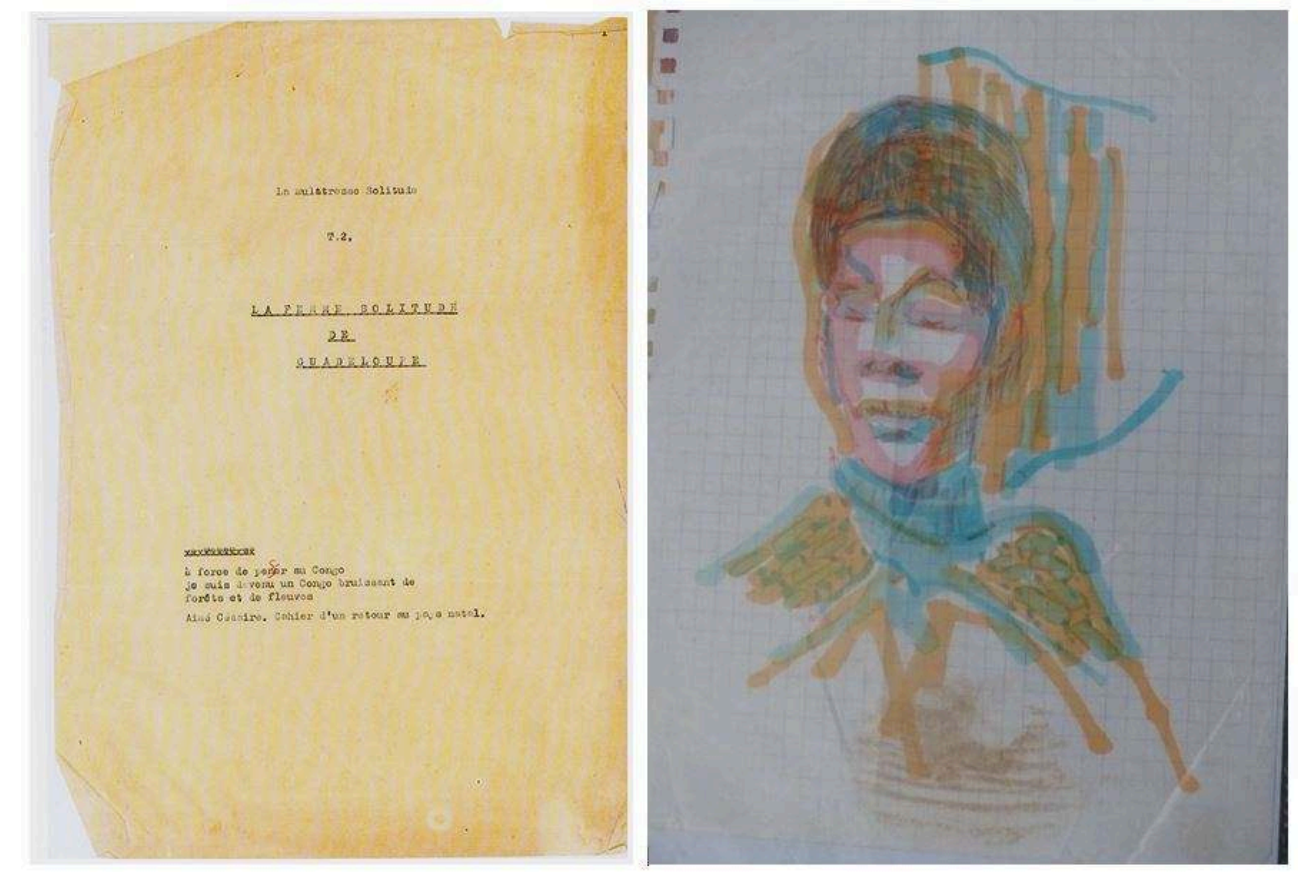

Bibliothèque nationale de France, fonds Simone et André Schwarz-Bart

3 L'avant-texte du roman est, à l'image de cette vacance des origines dont parle Simone Schwarz-Bart, éminemment fragmentaire. Le généticien dispose en tout et pour tout d'un manuscrit autographe - environ 150 pages de texte - correspondant à un stade avancé, voire très avancé puisqu'il est en partie calligraphié. Seuls les 48 premiers feuillets sont numérotés en continu, et l'écriture fine alterne ensuite avec les passages calligraphiés. Du point de vue du support, le texte a été rédigé sur des feuilles de classeur perforées à petits carreaux et sur la première page, l'encre a coagulé jusqu'à former la carte imaginaire d'une contrée lointaine et non répertoriée qui n'est pas sans évoquer la "planète étrange ${ }^{5}$ » $(\mathrm{MS}, 11)$ sur laquelle Bayangumay voit le jour sur cette même page (fig. 3). 
Fig. 3. Première page du manuscrit calligraphié du roman

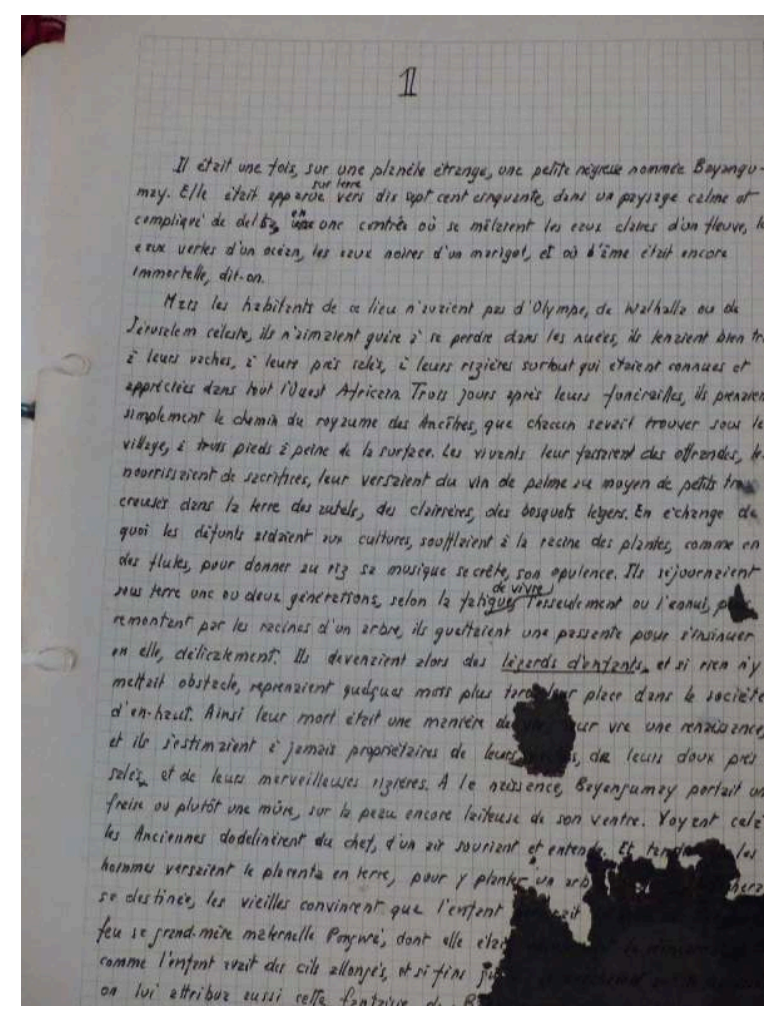

Bibliothèque nationale de France, fonds Simone et André Schwarz-Bart

4 À cette mise au net viennent s'ajouter des brouillons de plusieurs passages du roman, dont il est difficile de savoir s'il s'agit véritablement de premiers jets. Nous disposons en revanche de notes de travail à un stade beaucoup plus précoce de la composition du roman concernant l'épisode sur le négrier, d'autres sur la Révolution française aux Antilles, ou encore de versions antérieures de nombreux passages du roman, dont certaines pages rédigées ou dactylographiées sur du papier pelure ont tendance, parfois, à s'effriter 6 . Il y a enfin la précieuse correspondance de l'auteur, inédite également, avec ses éditeurs du Seuil ${ }^{7}$. N'oublions pas pour autant le paratexte publié : entretiens, dossier de presse, et bien sûr le texte de référence «Pourquoi j'ai écrit $L a$ mulâtresse Solitude ».

5 Il s'agit à présent de comprendre non plus seulement pourquoi mais aussi comment André Schwarz-Bart a écrit La Mulâtresse Solitude. Ce "petit livre »-comme l'auteur se plaisait parfois à l'appeler ${ }^{8}$ est en réalité le fruit d'une gestation longue et sinueuse, comme l'attestent les documents d'archives incontournables sur lesquels s'appuie cette étude, à savoir le manuscrit autographe du roman, les brouillons et les notes de travail afférentes, ainsi que la correspondance de l'auteur avec les éditions du Seuil. Grâce à ces documents inédits, il est possible d'identifier les étapes charnières et changements de cap qui ont jalonné cette genèse laborieuse. Il y a en particulier le moment où le roman éponyme se détache de l'écriture du cycle antillais pour constituer un ouvrage clos sur lui-même. Ou encore le déclic produit par la lecture d'historiens de la Guadeloupe tel Henri Bangou. Rares sont toutefois les sources décelables dans l'avanttexte, ce qui rend problématique le concept même d'exogenèse ${ }^{9}$. Partant de là, la présente étude tentera de montrer ce que l'avant-texte, si lacunaire soit-il, nous laisse entrevoir du projet initial et les traces - parfois spectrales - qui subsistent dans le texte 
publié. Cet examen à la fois macro- et micro-génétique permettra de suivre l'évolution et les mutations du projet esthétique d'André Schwarz-Bart au fil de la composition de l'œuvre.

\section{La « porteuse du temps »: Genèse et fluctuations du cycle}

Il semble opportun de revenir, même brièvement, sur le projet initial du cycle antillais, pour voir comment le texte qui nous intéresse ici tout à la fois s'y intègre et s'en émancipe. Car avant d'être le roman paru sous ce nom en 1972, La Mulâtresse Solitude est un cycle romanesque dont l'idée originelle remonte à 1955. Initialement conçu en 7 volumes, ce cycle aurait fait l'objet de 19 tentatives entre 1960 et 1965 et totalisé jusqu'à 1800-1900 pages, comme l'auteur le révèle dans un article du Figaro littéraire ( $n^{\circ}$ 1084) paru le 26 janvier 1967, texte séminal s'il en est intitulé « Pourquoi j'ai écrit $L a$ mulâtresse Solitude. André Schwarz-Bart s'explique sur huit ans de silence ${ }^{10} »$. Le titre fait référence au cycle et non encore au roman, lequel s'intitule alors «Bayangumay et la femme Solitude de Guadeloupe ${ }^{11} »$.

7 L'écrivain y relate que dès 1955 le projet d'un roman sur la condition noire a germé en lui, alors qu'il travaille depuis un an sur Le Dernier des Justes et fréquente les milieux antillais de Paris ${ }^{12}$. Il imagine son hérö̈ne, une jeune Antillaise née à Paris qui vivote dans une chambre de bonne avec sa grand-mère, et « ce qui [1]e hantait principalement, c'était une scène où la grand-mère s'efforce de raconter le pays à la jeune fille, laquelle n'a jamais vu la Martinique autrement qu'en songe» (HL, 9). Toutefois, ce n'est qu'au début des années soixante qu'il se jette dans l'écriture du cycle noir. Non seulement il n'y a pas de genèse imbriquée de La Mulâtresse Solitude avec Le Dernier des Justes, mais les documents de genèse révèlent plutôt la volonté de s'affranchir du choix esthétique du Dernier des Justes en faisant passer « la grand-mère, la vieille Martiniquaise, au premier plan» (HL, 12). L'auteur explique que petit à petit, la vieille femme prend vie dans sa tête et sous sa plume jusqu'à devenir une "porteuse du Temps» (HL, 12). Plusieurs lettres autographes inédites, rédigées entre 1961 et 1965, documentent ce momentcharnière dans l'évolution du projet esthétique du cycle, que Schwarz-Bart lui-même appelle sa "nouvelle conception». Parmi elles, quatre méritent une attention toute particulière $e^{13}$.

8 Avant l'installation du couple aux Antilles, le séjour au Sénégal de 1961 aura été déterminant en ce sens que celle qu'il appelle désormais " [sa] vieille négresse » (la future Mariotte) devient l'héroïne du cycle, rien de moins. Depuis Dakar, en août 1961, André Schwarz-Bart écrit à Paul Henri Flamand :

[...] pour l'instant j'habite davantage la grossesse de ma femme que le territoire africain. J'habite aussi un peu ma vieille négresse qui n'existe pas, et que pourtant j'imagine parfois, avec une précision angoissante, au détour d'une rue du vieux Dakar qu'elle aurait pu hanter il y a vingt-cinq ans ${ }^{14}$.

Mais surtout, dans une autre lettre datant probablement de fin 1961 ou début 1962, il explique qu'il fait fausse route en cherchant à calquer le cycle antillais sur Le Dernier des Justes :

La conception initiale était artificielle, en ce sens que j'utilisai pour les noirs le principe généalogique du "Dernier des Justes". La vie antillaise m'a conduit à une conception qui, tout en rendant compte de l'histoire du peuple noir, se fonde tout 
entière sur un seul personnage ; en l'occurrence une vieille négresse, une sorte de porteuse du temps dont le regard est tout un monde et par là même peut éclairer le monde tout entier ${ }^{15}$.

10 Cette bifurcation esthétique représente néanmoins une pierre d'achoppement, comme l'auteur le déplore dans une autre lettre : «En effet, il me faut rentrer car je suis devant la nouvelle conception comme devant une muraille infranchissable ${ }^{16}$. $"$ Et de Suisse, où il réside plus tard, il confie à son éditeur et à l'épouse de celui-ci : « Ça a été 2 volumes puis 3 et finalement je suis revenu à ces terribles 1800 pages (1 900 ?) autour desquelles je tourne depuis des années. Après, la chance a tourné et j'ai trouvé il y a 2 mois environ une conception que je crois simple, "musicale"... ${ }^{17}$ ".

11 C'est cette oscillation entre un et plusieurs continents, entre une et plusieurs voix, que l'auteur appellera rétrospectivement «l'accordéon » (HL, 16). Il envisage d'abord une version en trois tomes (Amérique, Afrique, Europe), puis il est « repris par la volonté esthétique d'exprimer tout le chant d'une seule voix » (HL, 16), le texte est donc réduit à un seul volume, mais l'Afrique n'occupe plus assez de place. Et ainsi de suite. Parallèlement, il échafaude son œuvre tout entière comme un triptyque (soit neuf volumes au total), dans lequel le cycle de La Mulâtresse Solitude - qui en constituerait le panneau central - serait encadré par deux romans juifs, respectivement Le Dernier des Justes et En souvenir du vingtième siècle ou le Récit de Moritz Lévy; mais lui-même qualifie son projet d'« improbable » (HL, 18). Les 1800 pages couvrent l'ensemble du cycle dont six volumes sont déjà à un stade avancé de la rédaction, mais il estime n'avoir pas encore trouvé le ton juste. C'est, selon les mots d'André Schwarz-Bart, l'intervention de Simone en 1964 qui mettra un terme à ces circonvolutions pour aboutir trois ans plus tard au roman à quatre mains que l'on connaît, premier du cycle.

\section{« Deux ou trois lignes que je sais d'elle » : Naissance d'une légende}

12 Du point de vue du roman, en revanche, le déclic se produit dès 1963, en Guadeloupe. Si la genèse du roman est enchevêtrée dans celle du cycle, La Mulâtresse Solitude n'en est pas moins un ouvrage clos sur lui-même, avec un statut particulier. Sa naissance coïncide avec le moment où la "nouvelle conception " - consistant à faire reposer le cycle tout entier sur un seul personnage - représente pour l'auteur un écueil (la «muraille infranchissable » dont il était question précédemment). En effet, il n'arrive pas à « établir d'une façon compatible avec la réalité historique, et en accord avec les lois de la vérité romanesque [...], la fameuse filiation généalogique qui permettrait de remonter au-delà de l'esclavage, jusqu'à l'Afrique pré-coloniale » (HL, 16). Cette fois, la difficulté ne concerne plus l'Afrique, ni même l'esclavage, mais la question de la transmission : "Si l'héroïne devait avoir connaissance [...] de sa filiation antillaise, il fallait que l'un quelconque de ses ancêtres esclaves ait défrayé la chronique » (HL, 21). Une fois ce principe établi, l'auteur se documente et c'est en lisant La Guadeloupe d'Henri Bangou ${ }^{18}$ qu'il découvre celle qui deviendra l'héroïne du tome deux du cycle. De fin 1963 à fin 1966, « Bayangumay et la femme Solitude de Guadeloupe » prend forme, non plus seulement dans sa tête mais sous sa plume.

13 Il s'écoulera, entre la publication d'Un plat de porc aux bananes vertes et celle de La Mulâtresse Solitude, cinq ans pendant lesquels une nouvelle bifurcation se révélera être déterminante pour la genèse du roman : l'arrêt de la collaboration avec Simone. C'est 
en 1970 que les deux écrivains prennent des voies - et des voix - divergentes, chacun écrivant son propre roman en parallèle (Pluie et Vent sur Télumée miracle et La Mulâtresse Solitude paraîtront la même année 1972). Dans un projet non utilisé de préface au cycle de La Mulâtresse Solitude, André analyse la fin de l'écriture collaborative en ces termes :

Après la parution, ayant pris un peu de recul, nous vîmes que nos voix se croisaient, se recouvraient parfois, mais ne se confondaient jamais. Nous essayâmes encore trois ans, puis ce fut l'abandon. Malheureusement, je m'étais attaché à des personnages, que je côtoyais depuis 10 ans, et pour certains quinze. Ne pouvant les abandonner tout à fait, je me résignai à un livre qui raconterait simplement une histoire avec ma voix : qui ne serait plus un témoignage mais une sorte de conte pour adultes $[. . .]^{19}$.

Fig. 4. Extrait du projet de préface au cycle de La Mulâtresse Solitude

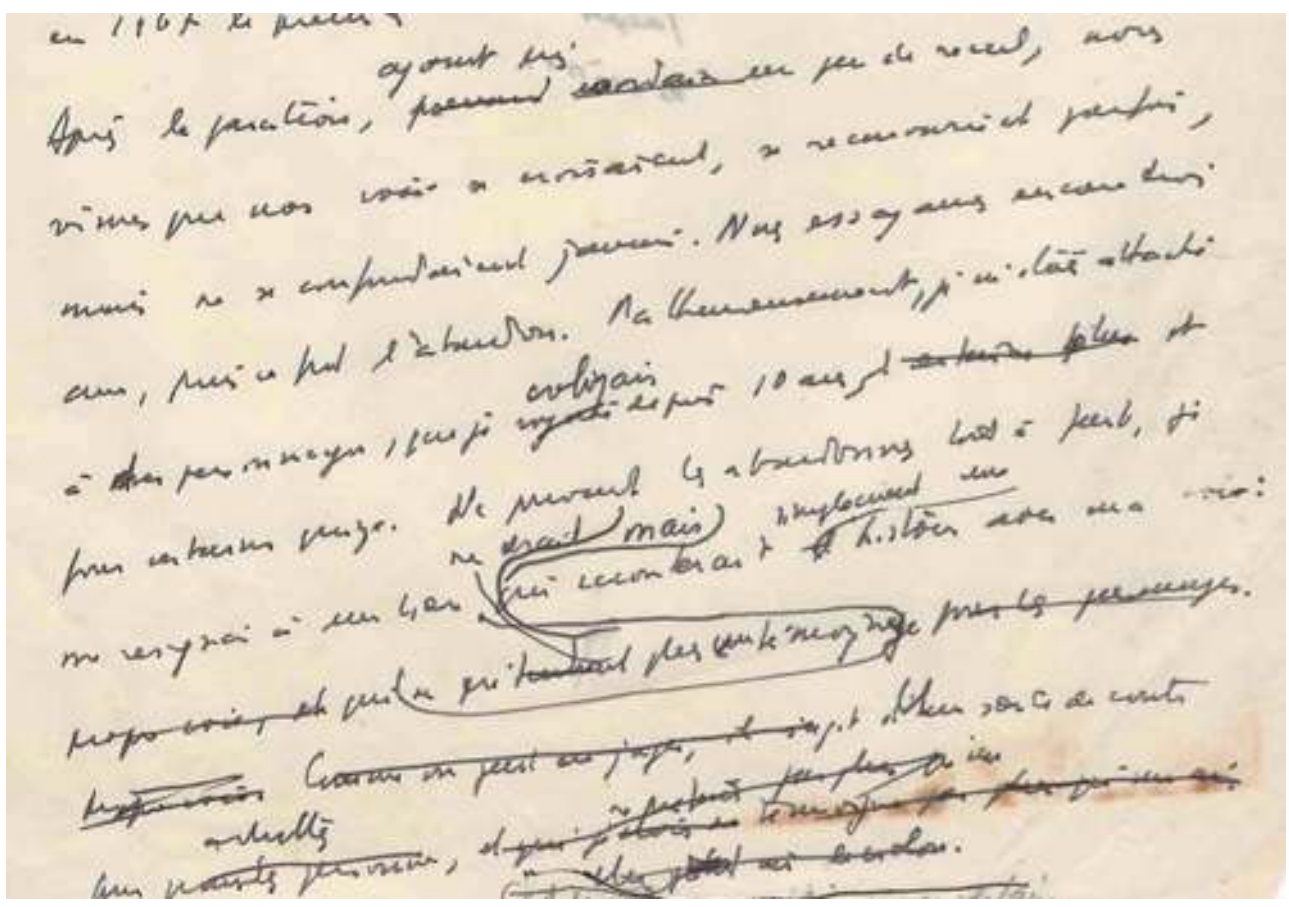

Bibliothèque nationale de France, fonds Simone et André Schwarz-Bart

L'avant-texte nous révèle donc deux choses. Tout d'abord, le roman à venir fournit à l'auteur, en la personne de Solitude - ancêtre illustre et rebelle, ancienne esclave devenue presque malgré elle l'égérie de la révolution en Guadeloupe aux côtés de Delgrès - la solution à ce qui représente alors pour lui une pierre d'achoppement, à savoir la question de la transmission, et ce dès 1963. Par ailleurs, plus tardivement sans doute vers 1970 et la fin de l'écriture à quatre mains - une évolution notable par rapport au projet initial se fait jour au sens où le «petit livre » change de nature : d'un roman-témoignage à la coloration épique, il dérive vers un portrait de femme plus intimiste aux allures de conte ou de légende. On voit ici les prémices de cette lignée de figures féminines fortes qui porteront le cycle dans son entier.

Soit dit en passant, cela représente un écart substantiel, une rupture même, par rapport au Dernier des Justes que Francine Kaufmann qualifie à juste titre de «saga identitaire ${ }^{20}$ ». Car si elle symbolise bien «la destinée d'une race ${ }^{21}$ ", Solitude incarne également, en tant que figure de proue de la résistance noire, la " terrible exception ${ }^{22}$ ", celle-là même que l'auteur s'interdisait de dépeindre dans son roman juif. Si paradoxal 
que cela puisse paraître, l'analogie pour le moins percutante qui clôt le roman, entre Delgrès et son armée improvisée morts en héros et les résistants juifs dont les âmes errent encore "parmi les ruines humiliées du Ghetto de Varsovie » (MS, 156), ne fait que creuser l'écart entre la légende de Solitude et l'histoire d'un héros juif ordinaire. On en revient une fois encore à cette béance des origines qui caractérise la culture antillaise. Dans une note de travail, Schwarz-Bart écrit :

Pour la fin de la deuxième partie, évoquer la suite des événements et dire qu'il n'en reste rien : les révoltés ont été punis jusque dans la mémoire des leurs, et il n'y a pas de monument à Delgrès - alors qu'il y a un monument au ghetto de Varsovie. Les Guadeloupéens ne connaissent pas la MS. Pas de monument dans leur mémoire. Il reste des chants, dont le sens s'est perdu $[. . .]^{23}$.

L'avant-texte corrobore ainsi, dans une large mesure, l'avant-propos de Simone Schwarz-Bart cité au début de cette étude. Ou du moins, il nous confirme que l'intention de l'auteur n'est pas tant de restituer une mémoire collective que d'en révéler l'absence.

Or, si le roman de Schwarz-Bart ne s'inscrit pas dans la tradition littéraire nordaméricaine du slave narrative ou récit d'esclave, c'est peut-être parce que dans les colonies antillaises, la parole des esclaves noirs a longtemps été oblitérée. Dans un projet de chapitre qui n'a pas été conservé dans le roman publié, l'auteur rapporte en effet que les registres relatifs aux procès d'esclaves étaient littéralement réduits en cendres, laissant aux seuls vainqueurs le soin de rédiger et perpétuer l'histoire officielle :

À de rares exceptions près, l'histoire a toujours été racontée par les vainqueurs; et l'une de ses fonctions traditionnelles, c'est de tuer une seconde fois les vaincus. Aux îles, tous les cinq ans, on avait coutume de brûler les minutes des procès d'esclaves relatifs aux esclaves. Hormis un bruit de légende, et quelques allusions effarées du notaire Vignot, rien ne demeure des paroles que prononça la mulâtresse Solitude devant ses juges. Nulle rue de Guadeloupe ne porte son nom. Elle n'est même pas devenue navire, comme le mulâtre Delgrès, qui deux fois par semaine fait le va et vient entre la Pointe à Pitre et lî̂le de St Barthélémy où il ramène un peu de viande sur pied. Nul camion de transport, nul char à passager, nulle coque bariolée de pêcheur ne porte le nom de Solitude. Nul enfant du pays. Il faut pour la trouver plonger dans quelque livre aux feuillets jaunis, où, généralement, elle apparaît sous un masque grimaçant ; ou bien dans les archives de l'ancien ministère des colonies, place du Trocadéro, Paris. En Guadeloupe, la seule trace qui demeure d'elle est une chanson, à laquelle manque, semble-t-il, un ou deux couplets ${ }^{24}$.

Ce texte mêlant fiction et réflexion personnelle est un véritable manifeste doublé d'un plaidoyer. Alors même que Solitude a contribué à écrire l'histoire collective guadeloupéenne, son existence (et partant, sa voix) a été niée car effacée des registres, pour être reléguée au rang de fait divers, de rumeur ou -expression quasi oxymorique - «bruit de légende ». Dans une perspective que l'on pourrait qualifier de postcoloniale, l'auteur extirpe son héroïne (au double sens du terme) de " quelque livre aux feuillets jaunis » au détour duquel elle est mentionnée sous un jour peu flatteur, pour la ramener à la vie, à défaut de lui rendre la parole. Le rire profond de Solitude lors des instants qui précèdent son exécution, ce « roucoulement léger, entrainant, à peine voilé de mélancolie» (MS, 152) n'apporte-t-il pas un démenti cinglant au «masque grimaçant» véhiculé par les historiens blancs, révisionnistes avant l'heure? Car l'ouvrage épinglé ici est sans nul doute celui d'Auguste Lacour, qui servira à Schwarz-Bart à la fois de référence et de repoussoir. Nous y reviendrons. 
Dans une note du 22 mars 1973, postérieure donc à la publication du roman, l'auteur renchérit :

En fait, je ne connais de Solitude que les deux ou trois lignes qui figurent dans d'obscurs manuels : (résumer la vie de Solitude en reprenant les termes de la vie du Christ). Et c'est pourquoi, désireux de la rappeler au souvenir des humains, j'ai donné à ma narration un caractère de légende $\mathrm{e}^{25}$.

Ces « deux ou trois lignes » sont celles placées en exergue de la première édition d'Un plat de porc aux bananes vertes, en guise de prélude au cycle tout entier. Elles proviennent des trois ouvrages sources (Bangou, Lacour et Oruno Lara) à partir desquels l'auteur a extrapolé pour retracer la « pauvre vie » (HL, 21) de Solitude et, sans entrer dans les détails, cette mise en exergue, déjà, est une réécriture ${ }^{26}$. La réappropriation des sources historiques nourrit l'invention romanesque au point de se confondre avec elle et la "pauvre vie " de Solitude revêt ainsi "un caractère de légende». Bien que rétrospective, la note ci-dessus a une valeur programmatique ( « résumer la vie de Solitude en reprenant les termes de la vie du Christ » sonne comme une injonction), alors même que l'auteur peine à définir le genre auquel appartient son «petit livre ». S'il parle dans le projet de préface au cycle d'une «sorte de conte pour adultes ", il donne ici l'impression d'hésiter entre hagiographie et légende - et les deux finissent d'ailleurs par se recouper puisque Solitude devient tout à la fois « héroïne et martyre » (HL, 21).

Dans le but à la fois d'étayer cette perspective macro-génétique et de mettre en lumière l'évolution de la conception du personnage de Solitude, nous allons à présent dériver vers quelques micro-analyses de passages tirés de l'avant-texte, certains précoces (entre 1963 et 1966) et d'autres plus tardifs (après 1970), respectivement les notes liminaires pour l'épisode sur le négrier et les brouillons du dernier chapitre du roman. Tous ont été choisis car ils en disent long sur la fabrique du récit et sur cette dialectique - qui fascine tant le généticien - entre l'intention de l'auteur et le jaillissement de l'imprévu.

\section{Au commencement était l'Afrique}

La Guadeloupe d'Henri Bangou paraît en $1962^{27}$, avec la dédicace « aux humbles de mon pays » et un avertissement précisant que l'ouvrage n'est " pas d'un historien » mais axé sur la "réhabilitation » de la collectivité guadeloupéenne «marquée du sceau d'une singularité qui la rend irréductible à l'histoire d'autres collectivités quelles qu'elles soient». Bangou s'attache à montrer comment l'institution esclavagiste dépasse le simple cadre économique pour façonner les sociétés des pays concernés. Au-delà du flot d'informations qu'il a puisées dans cet ouvrage remarquable, Schwarz-Bart s'est sans nul doute retrouvé dans une telle démarche, qui rejoint son projet humaniste. La fin de la première partie du roman et le début de la seconde sont émaillés de détails techniques tirés de toute évidence de l'ouvrage de Bangou, sur le cérémonial d'acquisition des esclaves suivi de l'estampage au fer chaud, ou encore les actes de révolte sur les navires négriers et les tentatives de suicide. On retiendra notamment le "mal de mâchoire », c'est-à-dire l'enfoncement dans la fontanelle de l'enfant d'une épingle sale au moment de l'accouchement, entraînant un tétanos mortel. Ces détails se retrouvent dans plusieurs notes de travail inédites d'André Schwarz-Bart. 
23 Six pages de notes programmatiques concernent le chapitre 3 de la première partie mettant en scène la capture puis le séjour sur le négrier, lequel figure le glissement vers la condition d'esclave. L'écriture est fine, avec très peu de ratures. Il s'agit de documents de travail, avec un savant mélange de réflexions personnelles, de notes à visée romanesque, de scénarios et de fragments de fiction. Bien qu'ils ne soient pas datés, nous pouvons les situer entre 1963 et $1966^{28}$, à un stade très précoce de la composition du roman où celle qui deviendra la mère de Solitude ne s'appelle pas encore Bayangumay mais Malicka, et l'enfant qu'elle engendre à la suite de la « pariade ${ }^{29}$ » est un garçon. L'on y découvre également que l'auteur prévoyait de mettre en scène une révolte sur le négrier (fig. 5 et 6). Ces notes précieuses sont à ce jour les traces les plus primitives du roman. À supposer que de nouveaux manuscrits ne viennent un jour invalider cette hypothèse, l'avant-texte nous révèle que la première partie du roman (Bayangumay) aurait été composée bien avant la deuxième partie (Solitude), ce qui du reste n'est guère surprenant. Car au commencement était le Sénégal. Au commencement étaient les Diolas :

Attaques venues d'autres villages Diolas. Arrivée au fleuve. Débarquement devant Ziguinchor où d'autres arabes et un homme rose, montés sur des bêtes étranges (chevaux) commandent d'autres files. On leur colle des bouts de bois. Puis une marche durant des jours, au cours desquels d'autres files étrangères viennent se mêler. Le pays change d'aspect. Suicides. Surtout des couples qui se suicident, comme par mode. Ceux qui faiblissent ont la tête tranchée pour récupérer le bois. Comme les gardiens violent les vierges, celles-ci le soir s'offrent aux esclaves ou fendent leur sexe de leurs ongles ou d'une pierre pour ne pas en laisser la primeur aux gardes ${ }^{30}$.

Le fragment ci-dessus se distingue par son caractère "scénarique " au sens où l'entend Pierre-Marc de Biasi : "centré sur un travail de planification, d'organisation et de structuration du récit, en grandes ou petites unités, sous la forme prospective de plans et de scénarios généraux ou partiels qui serviront de documents de régie ${ }^{31}$ ». De tels documents de travail correspondent très clairement à la phase durant laquelle l'auteur prend des notes destinées à alimenter les scènes et les personnages de son roman naissant, le plus souvent sous forme de ce que lui-même appelle un «plan », ce qui lui permet à la fois de planter le décor et d'avoir une vision d'ensemble de l'épisode précédant l'arrivée sur le négrier. Toutefois, la dimension macro-génétique n'est pas incompatible avec le fait d'injecter quelques faits précis («ceux qui faiblissent ont la tête tranchée" etc.) qui sont le fruit de ses lectures. Et cette vue panoramique n'empêche pas non plus les incursions dans le domaine du "scriptural ${ }^{32}$ ", toujours pour reprendre la distinction opérée par de Biasi. Car si l'on se place à l'échelle microgénétique cette fois, l'auteur détaille certaines scènes qu'il rédige entièrement ou presque. C'est le cas d'un épisode mettant en scène le fameux "mal de mâchoire » évoqué plus haut. Malicka met au monde un garçon qu'elle ne parvient pas à accepter et une vieille femme des environs venue aider pour l'accouchement tue l'enfant: « La vieille regarde et ne dit rien, mais après l'accouchement Malicka a l'impression, mais est-ce un rêve? que la vieille a retiré l'épingle à cheveux et enfoncé dans l'arrière du crâne du garçon ${ }^{33}$. " Dans le roman publié, l'auteur restera plus évasif sur les actes à la fois de mutilation et de rébellion recensés dans ses notes préparatoires pour être (ou non) développés par la suite. 
Fig. 5 et 6 : Notes pour des épisodes qui seront par la suite supprimés, en particulier la révolte sur le négrier

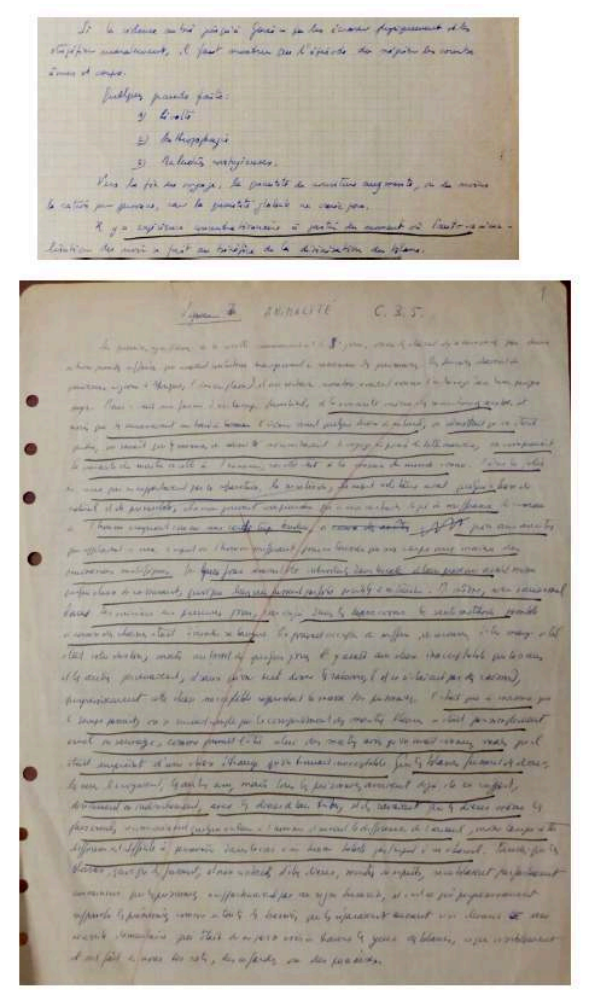

Bibliothèque nationale de France, fonds Simone et André Schwarz-Bart

Les fragments de fiction sont parfois entrecoupés de notes réflexives sur l'idéologie du système esclavagiste "concentrationnaire", parmi lesquelles ces deux phrases soulignées par l'auteur au feutre noir :

C'est par rapport à la divinisation du blanc que le séjour dans le négrier opère

l'entrée véritable dans le monde de l'esclavage [...].

Il y a expérience concentrationnaire à partir du moment où l'auto-animalisation du noir se fait au bénéfice de la divinisation du blanc ${ }^{34}:$ (fig. 5 )

On notera que sur le plan narratif, la stratégie choisie pour mettre en pratique cette idée est, déjà, le principe de la caméra subjective : «L'épisode doit être structuré en fonction de l'évolution interne de Malicka. Les faits ne doivent servir qu'à mettre en relief les mouvements de l'âme ${ }^{35}$.» Comme cela a été relevé, le nom de la protagoniste (Malicka) indique que l'auteur en est à un stade peu avancé de l'élaboration du roman. Or le principe de la caméra subjective va orienter la technique narrative du roman dans son entier - avec une héroïne qui a du mal à (se) verbaliser et un narrateur qui se fera intercesseur, ménageant des incursions dans la conscience du personnage. Ceci vaudra à la fois pour Bayangumay et pour sa fille Solitude, dans la version publiée. On se souvient par exemple de cette scène en contre-plongée où la première observe «trois hommes blancs » avec une curiosité mêlée de fascination, comme le trahit la présence de l'adverbe modalisateur « si »: « l'éclat de leurs dents si blanches entre les lèvres si rouges, tout cela dégageait un air d'indulgence lointaine, de bonté...» (MS, 39). Dans le manuscrit calligraphié, ces marchands d'esclaves étaient initialement "vêtus comme des dieux ${ }^{36}$ ", puis l'expression a été biffée et le lecteur passe sans transition de la naïveté bienveillante de la jeune fille à la sauvagerie extrême de ses bourreaux. L'étape 
suivante sera pour elle de ne plus se voir qu'à travers leur regard prédateur et dépréciateur.

Cette idée que les Noirs ne se voient plus que par reflet ou par réfraction au point de devenir de purs réceptacles de la pensée blanche a été surexploitée dans le roman, par le biais de deux métaphores filées déjà présentes dans l'avant-texte, et qui seront conservées avec des variantes. Il y a tout d'abord le fantasme de l'anthropophagie : le Blanc est perçu par le Noir comme un sorcier ou un vampire qui le vide de sa substance vitale, autrement dit lui dérobe son âme, avant d'asseoir son empire sur le monde. C'est une image centrale dans les notes sur le négrier. La seconde métaphore en est la suite logique : après avoir fait place nette, le Blanc pond ses œufs et se reproduit dans la tête du Noir jusqu'à lui instiller son idéologie délétère. Le Noir est alors captif, pris dans « la toile d'araignée de la pensée blanche ${ }^{37}$ » qui tisse des « fils invisibles ${ }^{38}$ » jusque dans son cerveau. Toutes ces métaphores feront leur chemin jusqu'au texte publié : jusqu'à Bayangumay dont "les hommes blancs, à la manière des sorciers, avaient sucé [l']intérieur » (MS, 43), puis jusqu'à Solitude qui se demande si elle ne se trouve pas «à l'intérieur du rêve des hommes blancs, comme tous ces pauvres nègres en uniforme dont la tête était pleine de larves pondues par les Blancs » (MS, 131).

Toujours sur l'intériorisation de la pensée blanche et l'autodénigrement du noir, il y a enfin cette note, selon moi capitale, exhumée et transcrite par Francine Kaufmann :

Je suis terrifié par l'imagination d'autrui, qui peut, d'un instant à l'autre, me faire entrer dans le champ de ses rêves, en m'y faisant jouer tel ou tel rôle à sa guise ; jusqu'à la mort, inclusivement. Les noirs (barré : juifs etc.) sont englués par les terribles pseudopodes de l'imagination des blancs. Nous avons beau nous débattre, refuser: l'imagination des blancs nous tient; nous sommes à l'intérieur de l'amibe ${ }^{39}$.

La biffure ( juifs ») est lourde de sens, puisqu'elle participe du principe de réversibilité qui sous-tend l'œuvre d'André Schwarz-Bart dans son entier. Mais c'est là une question tellement sensible, tellement intime, que l'auteur n'aura de cesse de tergiverser. Dans les notes de travail analysées précédemment, il arrive que le parallèle entre système esclavagiste et camp de la mort soit, au détour d'une comparaison, rendu palpable : "On prenait n'importe qui au départ, car il arrivait souvent que des nègres sains d'esprit et vigoureux de corps mouraient là où survivaient des fous et des débiles, comme dans les camps de concentration ${ }^{40}$.» Et à l'étape suivante, sur plusieurs tapuscrits, Schwarz-Bart ne titre plus « Négrier » mais « Déportation bateau ». Or dans la version publiée, il ne reste rien de ce parallèle sans doute trop facile aux yeux mêmes de l'auteur, ce qui rend d'autant plus percutante l'analogie finale déjà évoquée, entre l'habitation Danglemont et le ghetto de Varsovie. Le parallèle qui avait peut-être été tenté avec la Shoah dans une version primitive du roman s'estompe, et le ton du "sarcasme objectif, didactique ${ }^{41}$ " censé imprégner l'épisode sur le négrier cède progressivement la place à un mode onirique, fantasmagorique. Soit dit en passant, ceci vaut également pour la perspective historique: l'ironie voltairienne et le ton de la seconde partie annoncé dans l'avant-texte - «la révolution française en tant que joyeuse et sanglante comédie ${ }^{42} »-$ alternent avec le réel merveilleux, un peu comme dans Le Siècle des Lumières d'Alejo Carpentier, paru dix ans avant La Mulâtresse Solitude. Nous allons y venir.

30 Et pourtant, au fil du texte, le parallèle qui avait été éludé revient par des voies détournées, dans la phase finale de la composition du roman. L'exemple le plus spectaculaire de cette résurgence est « le lieu de l'holocauste » (MS, 147), en référence à 
l'habitation Danglemont où Delgrès se suicide avec ses hommes. Chose troublante, le mot « holocauste » ne figure pas encore dans la version calligraphiée du manuscrit du roman, pourtant tardive : il s'agit donc d'un ajout de dernière minute ${ }^{43}$. Comme si, loin d'être prémédité, le parallèle avait refait surface de manière inopinée au stade ultime de la rédaction. Ce jaillissement relève presque de "l'inconscient du texte ${ }^{44}$ ", selon une très belle formule que j'emprunte à Jean Bellemin-Noël. Nous terminerons cette étude par un autre surgissement peut-être plus inattendu encore, survenu également au stade ultime de l'écriture du roman : l'intrusion de la martyrologie chrétienne et d'une dimension christique.

\section{La Passion de Solitude, " cette sorte de chemin de croix »}

31 L'avant-texte de la seconde partie du roman se compose de documents assez hétérogènes: des notes sur l'époque révolutionnaire et ses protagonistes, en Guadeloupe mais également en Haïti dans une perspective comparatiste ${ }^{45}$; des « notes diverses » comme les qualifie l'auteur (souvent des fragments épars déjà rédigés); des notes réflexives, dont une datée du 10 février 1969 sur la perversité; des vestiges de la « vieille version » du roman; des brouillons de nombreux passages, dont certains, et non des moindres - la captivité puis l'exécution de Solitude - seront finalement supprimés ; ainsi que des portraits ciselés de nombreux personnages : les du Parc, Louis Mortier, le chevalier de Gallot (qui deviendra chevalier de Dangeau, et qui connaît, dans l'avant-texte, une mort tragique), Euphrosine Gellanbé, Rada (qui deviendra Maïmouni) ou encore la grande amie de Solitude Ti Soleil qui sera exclue du texte final. L'avanttexte laisse donc présager un récit agrémenté de faits et d'analyses, dans une veine historique classique. Le texte publié sera tout autre. 
Fig. 7. Notes sous forme de plan pour la deuxième partie

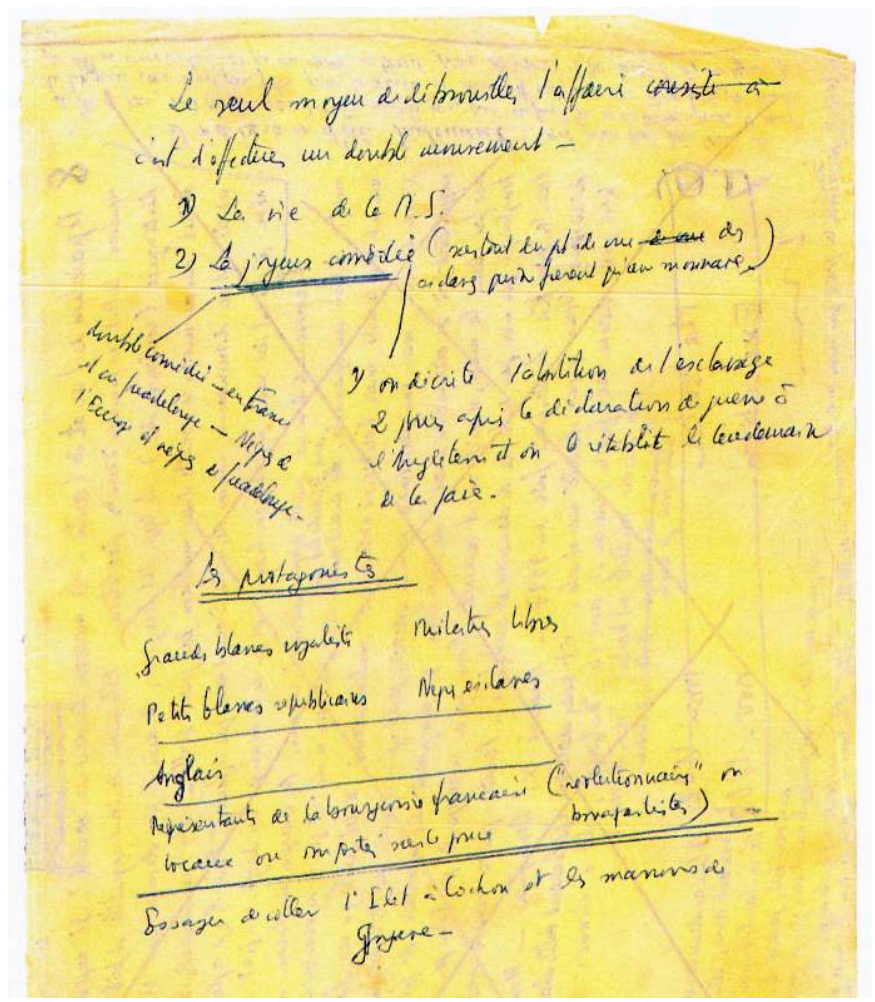

Bibliothèque nationale de France, fonds Simone et André Schwarz-Bart

En effet, hormis pour certains passages qui relèvent du conte philosophique, le texte publié de la seconde partie du roman dévolue à la courte vie de Solitude alterne entre le ton de la "chronique» (MS, 82) et celui du réalisme magique; ceci se confirme au chapitre 10 relatant la triste fin de l'héroïne. Ce revirement par rapport au projet initial est particulièrement perceptible dans un passage maintes fois retravaillé ${ }^{46}$ mettant en scène "l'apparition de la femme Solitude " (MS, 149) en route pour l'échafaud - en l'occurrence les trois derniers paragraphes du chapitre 10. Attardons-nous un peu sur cette scène de "supplice» (MS, 148) aux allures tout à la fois de procession religieuse, de carnaval et de cortège funèbre, pour la relire à la lumière des brouillons.

Il a déjà été relevé que le roman épouse en grande partie le point de vue des vaincus et si, pour l'auteur, cette stratégie narrative va de pair avec la tentative de réhabilitation d'une mémoire enfouie sous les décombres de l'histoire coloniale, elle ouvre également un espace de dialogue avec Lacour. Conseiller à la cour impériale, ce dernier fut aussi le premier historien blanc à mentionner la mulâtresse au tome III de son Histoire de la Guadeloupe, et son jugement est sans appel : «Solitude n'abandonna pas les rebelles et resta près d'eux, comme leur mauvais génie, pour les exciter aux plus grands forfaits ${ }^{47}$. Or, sur une feuille éparse, on découvre à notre grande surprise que celle qui deviendra un jour Mariotte dénonce « les traits de Furie que certains historiens blancs donnent à la mulâtresse Solitude, et surtout le Grand-Blanc M. A. Lacour, qui en fait une véritable tigresse assoiffée du sang des prisonnières blanches dévolues à sa garde, lors de l'affaire du camp de Dolé, en $1802^{48}$ ». Déjà dans l'avant-texte, certains passages sur Solitude sont imbriqués dans les souvenirs ou rêveries de sa (future) descendante: l'ombre furtive de la bisaïeule traverse ainsi le cycle antillais sous forme de rumeur ou de réminiscence. Comme si histoire et légende ne faisaient qu'un. 
Si Schwarz-Bart a puisé chez Lacour un flot d'informations précieuses, l'ouvrage lui aurait plutôt servi de repoussoir en termes de technique narrative. S'appuyant sur le récit des Blancs ou de leurs descendants qui furent faits prisonniers par les alliés de Palerme avant d'être épargnés, la démarche de Lacour relève de la tradition orale, mais du point de vue des vainqueurs. Schwarz-Bart, lui, adopte le point de vue adverse celui des Noirs, esclaves ou marrons ${ }^{49}$ - et à la fin du roman, le seul et unique passage dans lequel Solitude prend d'elle-même la parole pour déclamer « Fontaine... » (MS, 151) fait même l'objet d'un détournement ironique ${ }^{50}$. Au premier paragraphe de ce qui est devenu, dans le texte publié, une longue analepse, l'auteur nous donne à entendre le point de vue des Blancs sur Solitude de manière indirecte, par l'entremise du « notaire Vigneaux » (MS, 148), qui est en fait un avatar de Lacour ${ }^{51}$. On trouve en effet dans les brouillons correspondants quelques allusions à peine déguisées à l'historien : «[...] les témoignages affluaient dont fait encore état, un demi siècle [sic] plus tard, certain distingué historien de la Guadeloupe ${ }^{52}$ » (fig. 8). Mais toujours dans le but de conter l'histoire du point de vue des vaincus, le texte ira dans le sens d'une ellipse de la voix narrative historienne ou historicisante. Cet effacement s'accompagne d'une bifurcation esthétique majeure : de roman-témoignage, le récit dérive vers un portrait de femme. L'avant-texte recèle plusieurs exemples palpables de ce glissement, par exemple de nombreuses pages autographes dans lesquelles l'auteur semble vouloir calligraphier l'ensemble de son texte, en une version qu'il voudrait finale mais qui donne lieu à de multiples réécritures et donc de nouveaux brouillons.

Fig. 8 et 9 : Brouillons de la scène de supplice
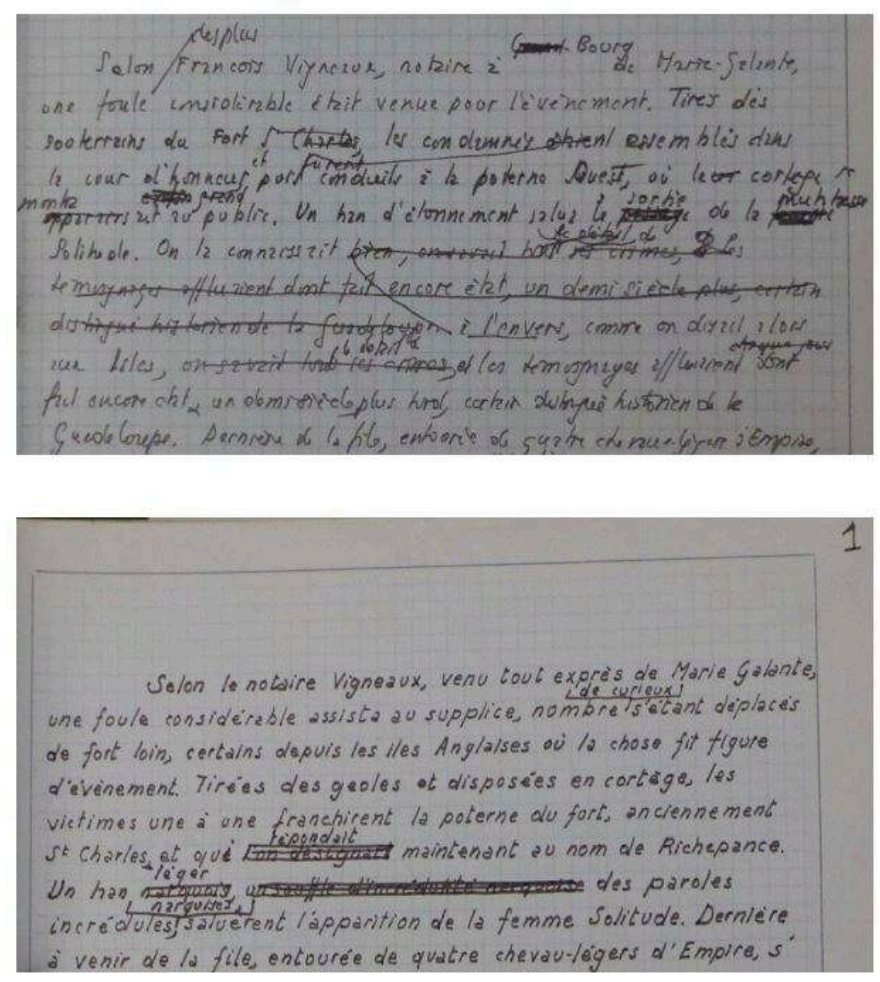

Bibliothèque nationale de France, fonds Simone et André Schwarz-Bart

Après avoir hésité entre "mulâtresse » et «femme Solitude » (fig. 8), la seconde finit par l'emporter comme en écho au titre originel du roman; puis le mot «apparition » 
(MS, 149) à connotation quasi flaubertienne supplante celui de "sortie " (lui-même venu en remplacement de "passage »), conférant à la scène un caractère évanescent (fig. 9). Et de fait, au second paragraphe, le récit glisse de manière subtile, par le biais d'une synesthésie, vers une mise en image de tout ce qui entre dans le champ de vision de l'héroïne et s'imprime sur sa rétine. Éblouie aux deux sens du terme, celle-ci flotte dans un état de semi-conscience et, selon la logique propre au rêve (mais peut-être aussi aux instants qui précèdent la mort), l'association d'idées remplace peu à peu la chaîne de causalité. Plusieurs séquences à valeur explicative seront ainsi supprimées du texte publié, notamment celle du " délivre » : la marche de Solitude jusqu'à l'échafaud survient au lendemain de son accouchement et, dans une scène qui sera expurgée, celle-ci redoute et essaie de retarder l'expulsion du placenta (ou « délivre»), d'où sa « désarticulation du plus étrange effet» (MS, 149) restée inexpliquée dans la version publiée. C'est encore le cas de l'analogie avec la Passion du Christ, qui surgit dans l'avant-texte de façon inattendue :

Quand elle s'avança vers la borne fontaine, la femme Solitude remarqua que la foule s'éloignait de plusieurs pas à son approche ; au lieu que, tout à l'heure, au début de cette sorte de chemin de croix (selon l'expression curieuse du notaire Vigneaux) les blancs avaient tendance à rompre le barrage devant elle, comme pour lui lancer de plus près leurs insultes et ces molles pommes cannelles qui giclaient, au contact de sa robe... ${ }^{53}$

L'analogie disparaît du texte publié - et pour cause, elle véhicule le point de vue du notable blanc - et le calvaire de Solitude revêt finalement « un petit air de fête » (MS, 150) puisque la scène nous parvient à travers le regard émerveillé de celle-ci.

Parmi les autres scènes qui seront supprimées se trouve une page magnifique. Observant celui qui est sur le point de l'exécuter, l'héroïne a soudain une révélation une épiphanie - dans un sens mystique : «c'est l'intuition éblouissante de l'enfant que fut cet homme, et qu'il demeure, encore ", et elle entrevoit «la voie mystérieuse qui conduisit un petit paysan blanc d'une douzaine d'années, à ce chien de garde humain recru de peurs anciennes enfouies sous le mot devoir... ${ }^{54}$ ». Dans la version publiée, en lieu et place de l'empathie christique que Solitude ressent pour son bourreau, c'est une "négresse à madras " (MS, 151) qui, comme sous l'effet d'un prisme déformant, en vient à éprouver de la miséricorde pour la suppliciée et dépose à ses pieds « une herbe dite de l'enfant Jésus » (MS, 150) en guise d'offrande. Hagiographie ${ }^{55}$ et légende se rejoignent enfin et le texte se donne à lire comme un récit de vie aux allures de fable issu de la tradition populaire. C'est là, en ce dernier matin, que Solitude « sembl[e] extraire sa force d'âme du cœur même de sa faiblesse, de sa fragilité, de son infinie vulnérabilité ${ }^{56} »-$ au plus loin de la harpie vengeresse décriée par Lacour. Ainsi, au fil des réécritures successives du tableau final, l'hostilité de la foule venue assister à l'exécution de Solitude sera atténuée pour se muer en compassion, accentuant de ce fait sa condition de martyre - même si elle est une martyre insoumise. Dans le même temps, moult détails seront supprimés (par exemple la biographie condensée de ladite «négresse à madras ») parce que, conformément au principe de la caméra subjective, le lecteur ne doit pas en (sa)voir plus que l'héroïne. Mais surtout, parce qu'il prive le lecteur d'autant d'éléments explicatifs, le texte publié entretient le mystère à son endroit. La « légende » de Solitude est à prendre au sens fort du terme : elle doit être lue, partagée à voix haute à la manière des "récits de veillée » (MS, 152) et, comme toute légende, elle se doit de conserver quelques zones d'ombre. Enfin, tout ce qui 
pourrait faire dévier le récit du côté de la fresque historique est là encore supplanté par un mode onirique qui frôle parfois le réel merveilleux.

Revenons une dernière fois sur le combat de Solitude aux côtés de Delgrès. Les notes de Schwarz-Bart sur Delgrès sont un cas d'école ${ }^{57}$. Elles sont largement fictionnalisées et les sources historiques (Bangou, Lacour) y sont devenues quasi méconnaissables, repoussant toujours plus loin les frontières - déjà mal définies - de l'exogenèse. Le texte publié, comme d'ailleurs l'avant-texte, précise que Solitude fut «blessée à la tempe » (MS, 147) : c'est là un détail sans aucun fondement historique, qui a pour effet de souligner la fragilité de l'héroïne, sa fêlure au sens propre du terme. Et de fait, il s'agit d'une référence exclusivement endogénétique qui renvoie à plusieurs passages de l'avant-texte, expurgés par la suite. Nous en retiendrons deux. Tandis que Solitude s'encanaille, errant dans les rues de Pointe-à-Pitre au lendemain de l'abolition de l'esclavage, une explication rationnelle nous est fournie au détour d'un portrait sans concession qui fait ressortir sa facette froide et calculatrice, laquelle sera finalement évincée :

On la retrouve encore très belle, ensauvagée de rhum, dans les petits cafés de la place Untel où ripaille une faune de corsaires internationaux, d'émissaires de la République, etc... Elle est extrêmement dépensière, etc... Elle vit avec Untel et Untel, etc... Mais si de sang-froid elle est à la fois parfaitement luxurieuse et servile, le fonds de l'ivresse lui imprime une sorte de détente, - comme aux pieds de celui qui se noie le fond de l'eau - la rend sarcastique, impertinente; un jour elle reçoit un coup de crosse qui lui marque la tempe - vilainement ${ }^{58}$.

Fig. 10 : Passage extrait de plusieurs pages sur la « joyeuse comédie » de la Révolution

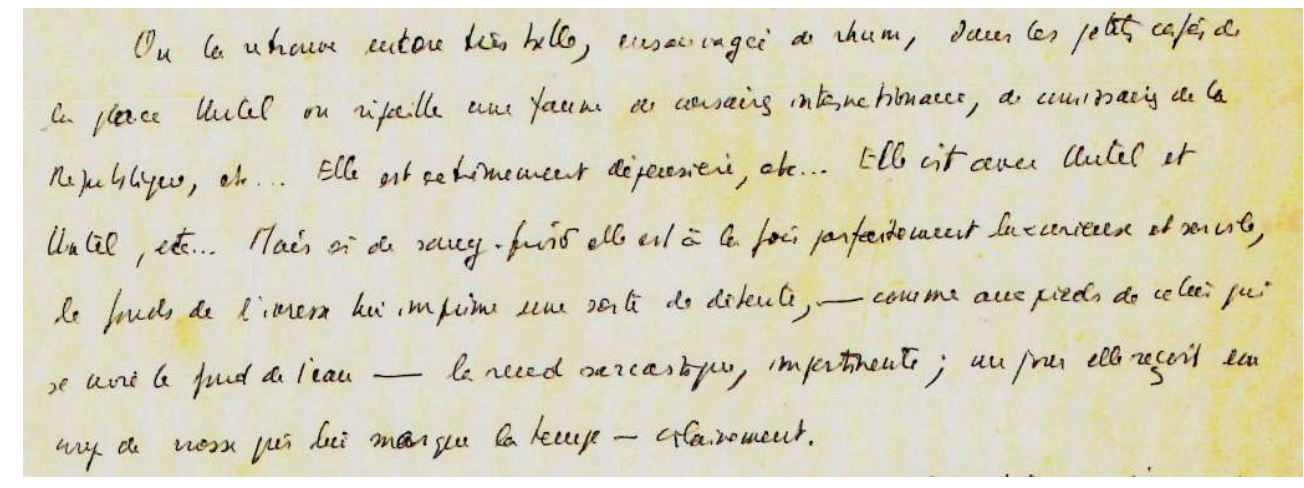

Bibliothèque nationale de France, fonds Simone et André Schwarz-Bart

Incarcérée au fort Saint-Charles dans un brouillon ultérieur, elle est enchainée comme jadis sa mère sur le négrier - et les badauds défilent nombreux pour ausculter cette bête de foire. Mais elle est pour ainsi dire dans les limbes, comme si son âme était prête à s'envoler vers l'Afrique. Et c'est là que jaillit dans l'avant-texte la métaphore sublime du «vol des pensées de Solitude», qui nous conduit aux confins du réel merveilleux :

Solitude n'avait pas encore claire conscience du monde. Sa tempe cassée libérait les sources de son esprit, et un flot de pensées merveilleuses s'en échappait, de jour et de nuit. Autrefois, ses pensées creusaient des trous de taupe, au fond desquels elle se glissait, se perdait, s'ensevelissait. Mais depuis que sa tempe était cassée, elles pointaient leur bec dans la fissure, comme des oiseaux de roche, prenaient leur envol, s'élevaient dans les airs avec des grâces, des caprices, des volte-face étonnantes. Ainsi, plus rien ne s'opposait à ce qu'elle que Solitude prenne le bateau pour l'île à Congo $[. . .]^{59}$. 


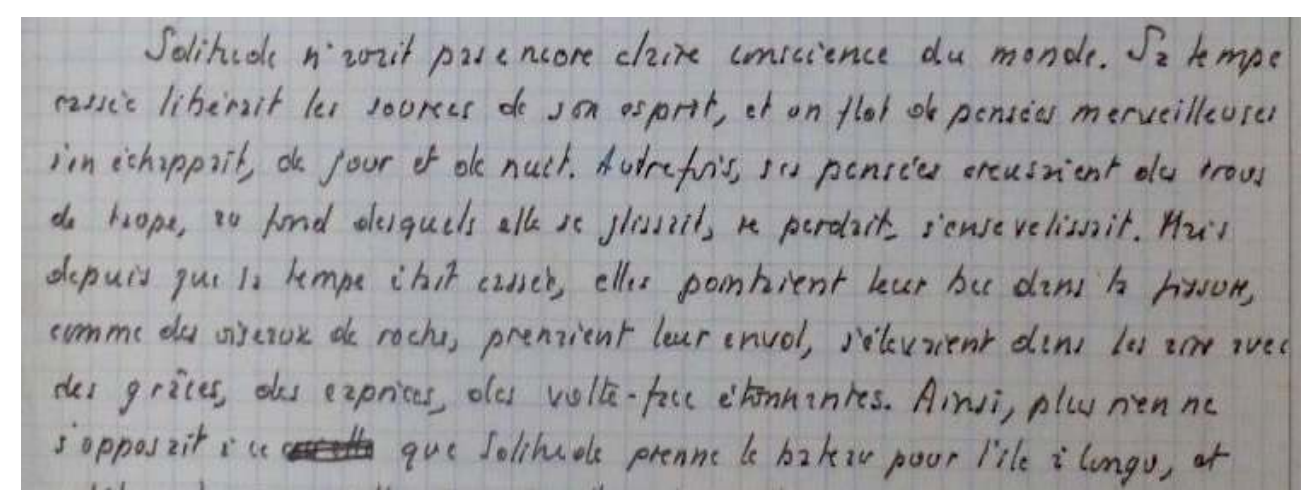

Bibliothèque nationale de France, fonds Simone et André Schwarz-Bart

40 On voit bien, d'un brouillon à l'autre, le changement radical de ton et de registre. Les deux tonalités alterneront dans le texte publié et, si elles se télescopent parfois, l'ironie voltairienne sera réservée pour l'arrière-plan historique et la «comédie» de la Révolution, tandis que prose poétique et réalisme magique viendront rehausser la triste légende de Solitude.

41 Pour conclure brièvement, l'avant-texte permet de lever le voile sur certains événements tragiques et emblématiques dont le texte publié ne donne qu'une version tronquée : la pariade sur le négrier suivie de l'infanticide et, à l'autre bout de la chaîne, la captivité puis l'exécution de Solitude. Le choix de retrancher ces éléments explicatifs participe sans nul doute du parti pris esthétique de tirer le récit du côté de la légende et du rêve - ce qui représente un écart notable par rapport au dessein initial d'écrire un roman à valeur de « témoignage ». Et de fait, le texte, qui aurait facilement pu dévier du côté de la fresque historique - comme le laissaient présager les notes de lecture sur la Révolution haïtienne et la Révolution française dans les colonies - dérive dans les brouillons vers un portrait de femme intimiste et un récit de vie. Mais il y aurait peutêtre, aussi, une seconde explication. Dans une conversation à bâtons rompus qu'il avait eue avec Francine Kaufmann en août 1972, soit quelques mois seulement après la sortie de La Mulâtresse Solitude, André Schwarz-Bart mettait en relation l'esclavage avec la Shoah en ces termes :

J'ai toujours ressenti qu'il y avait un péché à écrire sur l'Holocauste; d'où mes remords, mes nombreux repentirs d'écriture. Je pense que la seule réponse à l'événement est le silence. [...] Plus tard, conscient de mon échec, j'ai tenté d'exprimer le "silence" à travers une autre réalité, qui est la tragédie du monde noir ${ }^{60}$.

C'est pourquoi je serais tentée de mettre en rapport le retranchement ou l'omission de nombreux passages de l'avant-texte avec cette tentative impossible de restitution d'une mémoire collective pour laquelle il n'existe aucun témoignage direct de la part d'un peuple «mutilé, coupé, tranché de soi-même » $(\mathrm{HL}, 12)$. D’où, déjà, la tentation du silence et le renoncement. Le récit, comme la tempe de l'héroïne, se "fissure", se troue, se perfore à mesure qu'il s'écrit. Et l'on sait à présent quelle expression a été biffée, quel épisode élagué, quel personnage évincé - comme autant de béances qui viennent encore hanter le roman publié. À la lumière de sa genèse, ce " petit livre » que la publication avait comme figé dans l'ambre s'anime et prend une tout autre dimension. Il se révèle (à) lui-même. Et c'est comme si toutes ces matrices de textes, ces 
potentialités inassouvies ou inexploitées contenues dans l'avant-texte s'imprimaient en négatif dans le texte publié, que l'on connaît pourtant bien mais que l'on relit à présent avec un regard neuf.

\section{NOTES}

1. Lettre d'André Schwarz-Bart à Susan Dunne, non datée, en réponse à une lettre de cette dernière du 21/01/2003. BnF, fonds Schwarz-Bart. Cette aspirante scénariste avait envoyé un courrier aux époux Schwarz-Bart pour s'enquérir des droits du roman La Mulâtresse Solitude.

2. André SCHWARZ-BART, La Mulâtresse Solitude, Paris, Seuil, 1972.

3. Simone et André SCHWARZ-BART, L'Ancêtre en Solitude, Paris, Seuil, 2015, p. 12.

4. Toutes les illustrations proviennent du fonds Simone et André Schwarz-Bart de la Bibliothèque nationale de France. L'accès au fonds et au dossier génétique de La Mulâtresse Solitude m'a été grandement facilité par Jérôme Villeminoz, conservateur du fonds Schwarz-Bart au département des Manuscrits. Qu'il en soit remercié ici très chaleureusement.

5. On utilisera l'abréviation MS pour toutes les citations tirées du roman.

6. Ces documents font partie du fonds Schwarz-Bart qui se trouve à la Bibliothèque nationale de France (BnF), site Richelieu.

7. La correspondance d'André Schwarz-Bart avec Paul-André Lesort et Paul Henri Flamand respectivement fait partie des archives du Seuil conservées à l'Institut Mémoires de l'édition contemporaine (IMEC), abbaye d'Ardenne. Si le premier (PAL) était l'éditeur de Schwarz-Bart au sens fort du terme (c'est avec lui que l'auteur traitait pour le suivi du manuscrit du roman et les corrections), Flamand - qui était président-directeur général du Seuil - fut lui aussi très impliqué dans toutes les étapes de la création du cycle et du roman, au sens où l'auteur lui faisait part très régulièrement de l'avancée de son projet.

8. Lettre d'André Schwarz-Bart à Susan Dunne citée en exergue.

9. Pour rappel, et comme l'explique très bien Pierre-Marc de Biasi, « l'exogenèse ne désigne pas les "sources" de l'œuvre (telle personne réelle, tel lieu, telle œuvre littéraire), mais la trace repérable de ces référents-sources en termes de documents (écrits ou transposés) qui se trouvent attestés dans le dossier de genèse ». Pierre-Marc DE BIASI, «Qu'est-ce qu'un brouillon? Le cas Flaubert: Essai de typologie fonctionnelle des documents de genèse », dans Pourquoi la critique génétique: méthodes, théories, Michel cONTAT et Daniel FERRER (éd.), Paris, CNRS Éditions, 1998, p. 47-48.

10. L'article en question a été publié au Seuil (1967, édition hors commerce) sous le titre Histoire d'un livre: La mulâtresse Solitude. Lorsqu'il est cité ici, la pagination renvoie à l'édition du Seuil, avec l'abréviation HL.

11. Dans son article indispensable «Le projet judéo-noir d'André Schwarz-Bart: saga réversible ", Francine Kaufmann explique que le premier volume du cycle antillais, remis à l'éditeur en novembre 1966, était signé des noms associés de Simone et d'André et qu'en regard de la page de titre, plusieurs ouvrages en préparation étaient annoncés. De Simone seule, Pluie et Vent sur Télumée Miracle. Et sous le titre global de La Mulâtresse Solitude, un cycle de sept volumes à quatre mains, dont six en préparation, avec pour titres respectifs : Les livres et ma vie, Bayangumay et la femme Solitude de Guadeloupe, Martinique ho!, Bogota Bogota, Mère Afrique, Paris (suivi de 
l'épilogue). Francine KAUFMANN, «Le projet judéo-noir d'André Schwarz-Bart : saga réversible », Présence francophone, $\mathrm{n}^{\circ} 79$ (1), 2012, p. 22.

12. Le catalyseur aurait été une conversation de l'auteur avec une amie antillaise, empreinte de fatalisme ("dans cent ans, dans mille ans... une négresse restera toujours une négresse ») et celui-ci nourrit alors l'ambition « de lui montrer qu'elle se trompait [...] par un livre » (HL, 8).

13. Ces lettres s'étirent approximativement de 1961 à 1963-64. Étant pour la plupart non datées mais émaillées de repères biographiques, je remercie chaleureusement Francine Kaufmann d'avoir si généreusement déroulé son fil d'Ariane pour m'aiguiller dans ce labyrinthe.

14. Lettre à Paul Henri Flamand, août 1961. IMEC, SEL 3921.2.

15. Lettre manuscrite à Paul Henri Flamand, non datée. IMEC, SEL 3921.2. Expression soulignée par l'auteur.

16. Lettre manuscrite, non datée, en réponse à une lettre de Paul Henri Flamand de janvier 1963. IMEC, SEL 3921.2.

17. Lettre adressée depuis Prilly-Lausanne à Paul-André Lesort et sa femme, non datée. IMEC, SEL 3921.1. Francine Kaufmann m'a fait remarquer à juste titre que Simone y est mentionnée en tant que lectrice et non encore comme collaboratrice, elle date probablement donc de fin 1963-début 1964.

18. «Un jour, parcourant un ouvrage de mon ami Henri Bangou, historien de la Guadeloupe, je tombai par hasard sur l'épisode de la mulâtresse Solitude, héroïne et martyre de la grande révolte des esclaves qui ensanglanta la fin du XVIII e siècle. » $(\mathrm{HL}, 21)$

19. « Note" (recto). Note autographe, recto-verso, non datée, projet de préface au cycle de La Mulâtresse Solitude. BnF, fonds Schwarz-Bart. Transcription simplifiée Catherine Rovera.

20. Francine KAUfMANN, art. cit., p. 16.

21. Tels sont les mots d'André Schwarz-Bart : « C'est aussi l'histoire d'une femme qui symbolise la destinée d'une race » (extraits du résumé, par André Schwarz-Bart, de son roman en vue d'une adaptation cinématographique par Claude Faraldo et André Schwarz-Bart. BnF, fonds SchwarzBart).

22. Dans la seconde version du Dernier des Justes, André Schwarz-Bart explique qu'il a " essayé de créer un personnage exemplaire, un héros de roman qui serait un véritable juif de notre temps » et c'est pourquoi il n'a " pas cherché ce héros parmi les révoltés du ghetto de Varsovie, ni parmi les résistants qui furent, eux aussi, la terrible exception ». Liminaire du chapitre "La Légende des Justes », extrait de la « Biographie d'Ernie Lévy », publié dans la revue du FSJU de décembre 1956, p. 26, reproduit en fac-similé dans l'annexe de la thèse de Francine Kaufmann (Le Dernier des Justes d'André Schwarz-Bart : genèse, structure, signification, 1976, p. 404).

23. «La M.S. Deuxième partie ». Note de travail autographe (sous forme de plan), non datée. BnF, fonds Schwarz-Bart. Transcription Catherine Rovera.

24. «Ancien chap XI vieille version MS ». Page autographe, non datée. BnF, fonds Schwarz-Bart. Transcription Catherine Rovera. «Ces lignes étant tracées en 1970 » : ainsi commence le second paragraphe du chapitre XI, mais rien ne permet de cautionner la véracité de cette date, mentionnée au détour d'un texte qui relève avant tout de la fiction.

25. Note autographe. Jeudi 22.3.73. Archives Schwarz-Bart. Transcription simplifiée Catherine Rovera.

26. Dans le cas de Lacour par exemple, la mise en exergue repose sur le montage de différentes citations et l'ordre entre les phrases a parfois été inversé. Il est par ailleurs intéressant de relever que certaines des sources bibliographiques fournies par l'auteur à la fin de La Mulâtresse Solitude sont approximatives et les dates de publication parfois erronées, pour Bangou notamment.

27. Henri BANGOU, La Guadeloupe, 1492-1848 ou l'histoire de la colonisation de l'île liée à l'esclavage noir de ses débuts à sa disparition, Aurillac, Éditions du centre, 1962.

28. Schwarz-Bart lit Bangou en 1963 et fin 1966. Son roman s'intitule «Banyangumay et la femme Solitude de Guadeloupe », les notes se situent donc quelque part entre les deux. 
29. Le mot figure entre guillemets dans l'avant-texte. «C.3.6 ». Notes de travail autographes. BnF, fonds Schwarz-Bart.

30. « C.3.4» (PLAN SÉLÉKY). Note de travail autographe. BnF, fonds Schwarz-Bart. Transcription Catherine Rovera.

31. DE BIASI, art. cit., p. 50.

32. Ibid.

33. «Malicka Guadeloupe ». Page autographe, numérotée 9 par l'auteur. BnF, fonds SchwarzBart. Mot souligné par l'auteur. Transcription Catherine Rovera.

34. « C.3.4 » (PLAN SÉLÉKY). Note de travail autographe. BnF, fonds Schwarz-Bart.

35. « C.3.4 » (PLAN SÉLÉKY). Note de travail autographe. BnF, fonds Schwarz-Bart.

36. "La mulâtresse Solitude T.2. LA FEMME SOLITUDE DE GUADELOUPE." Manuscrit autographe, à la page 22 de la partie calligraphiée et paginée par l'auteur. BnF, fonds SchwarzBart.

37. « MS vieille version MS p. 117 ». Page autographe calligraphiée. BnF, fonds Schwarz-Bart.

38. Brouillons autographes de la seconde partie du roman, pages numérotées 7 et 8 par l'auteur. BnF, fonds Schwarz-Bart.

39. Note autographe. Archives Schwarz-Bart. Transcription Francine Kaufmann.

40. «C.3.6» [NÉGRIER]. Note de travail autographe. BnF, fonds Schwarz-Bart. Transcription Catherine Rovera.

41. «C.3.6 » [NÉGRIER]. Note de travail autographe. BnF, fonds Schwarz-Bart.

42. Note de travail autographe (sous forme de plan/projet), non datée, intitulée «La M.S. Deuxième partie » [BnF, fonds Schwarz-Bart]. La formule souligne l'absurdité du fait que l'esclavage ait été aboli après la déclaration de guerre à l'Angleterre puis rétabli huit ans plus tard au lendemain de la paix.

43. Une explication à ce rajout pour le moins surprenant pourrait être la lecture par SchwarzBart de L'Épopée Delgrès : La Guadeloupe sous la Révolution française (1789-1802) de Germain Saint-Ruf (Paris, Éditions Librairie de l'Étoile, 1965), où figure l'expression «l'holocauste de Delgrès au Matouba » (réédité chez L’Harmattan, 1977, p. 147). Si « holocauste » y est synonyme de sacrifice, ce terme permet à Schwarz-Bart de jouer sur la polysémie et de préparer l'analogie finale avec le ghetto de Varsovie. Faut-il y voir une pure coïncidence ou un emprunt distrait de la part d'un auteur qui avait pour habitude de distordre ses lectures au point de les rendre méconnaissables? En tout cas, rien dans l'avant-texte ne nous autorise à affirmer que Schwarz-Bart avait lu SaintRuf.

44. Jean BELLEMIN-NOËL, Vers l'inconscient du texte, Paris, PUF, 1979. Il y défend l'idée que si un texte ne s'écrit pas tout seul, l'écriture dépasse l'inconscient du sujet écrivant. Soit dit en passant, c'est également à Bellemin-Noël que l'on doit le concept fondamental d'«avant-texte » (Le Texte et l'Avant-texte, Paris, Larousse, 1972).

45. Au fil des notes prises par Schwarz-Bart sur la Révolution, on trouve des commentaires tels que : « [l]a révolution en Haïti triompha parce qu'elle avait un esclave noir à sa tête ; le sort de la révolution guadeloupéenne se joua entre les mains délicates de deux "grands-mulâtres", affranchis depuis toujours : Pélage et Delgrès ». Note autographe sur la Révolution. BnF, fonds Schwarz-Bart.

46. Peut-être Paul Henri Flamand fait-il allusion à ces derniers brouillons de l'ordre du ressassement, lorsqu'il écrit à son confrère chez Heinemann : «Le cas Schwarz-Bart est en quelque sorte tragique car il ne cesse de travailler, il a écrit des pages et des pages et peut-être, en effet, comme vous le dites la question est-elle pour lui "de finir". Et nous devons agir doucement tant est grande son angoisse, tant est farouche son recul et tels sont les ménagements dont nous devons user pour ne pas l'acculer à des actes inconsidérés. » Lettre de Paul Henri Flamand à Warburg, 16 mars 1971. IMEC, SEL 3921.2. 
47. M. A. LACOUR, Histoire de la Guadeloupe, vol. 3 « 1798 à 1803 », Basse-Terre, Imprimerie du gouvernement, 1858, p. 311. L'ouvrage est consultable sur le site gallica.bnf.fr.

48. Page autographe, sans titre ni date, paginée 16 par l'auteur. BnF, fonds Schwarz-Bart. Transcription Catherine Rovera.

49. Sur ce point, je trouve ce commentaire tiré d'une critique de La Mulâtresse Solitude particulièrement pertinent et perspicace : «Les personnages blancs n'existent à peu près pas dans le livre: ils se confondent avec les instruments de torture par lesquels ils se manifestent. Nous restons au niveau de la conscience des Noirs, ou au niveau de leur ignorance douloureuse » (Robert KANTERS, « Au-delà de toute solitude », Figaro littéraire, n 1343, 11 février 1972).

50. Chez Lacour, ce sont là les dernières paroles qu'au moment de la pendaison un maquignon adresse à ses compagnons d'infortune (LACOUR, op. cit., p. 340), alors qu'ici la mulâtresse, en toute insolence, interpelle le "parterre des Blancs » (MS, 151), comme sous l'effet d'un renversement carnavalesque.

51. Dans la "vieille version ", les paroles du notaire étaient rapportées au discours direct : «Le jour de son exécution, dit le notaire Vigneaux, la femme Solitude montra un visage chafouin, vieillot, tout en angles... etc. ». «Exécution MS vieille version», p. 133. Tapuscrit paginé par l'auteur (BnF). On signalera au passage que la simple orthographie du nom "Vigneaux " nous révèle que la scène a été écrite tardivement (car dans l'« Ancien chap XI » cité précédemment et ultérieur sans doute à 1970, le notable en question s'appelait « Vignot »).

52. Brouillon autographe. BnF, fonds Schwarz-Bart. Transcription simplifiée Catherine Rovera.

53. Brouillon autographe. BnF, fonds Schwarz-Bart. Transcription Catherine Rovera.

54. Tapuscrit, avec annotation autographe : «Le bourreau qui va exécuter Solitude est l'ancien enfant qu'il fut ». BnF, fonds Schwarz-Bart.

55. Solitude incarne la "terrible exception », et son exemplarité lui confère une aura, une forme de sainteté dans plusieurs passages où les connotations bibliques le disputent au réel merveilleux. Telle une sainte ou une miraculée, elle "entre jusqu'à mi-corps dans la rivière à Goyaves, le buste soumis à la caresse des grands arbres et les jambes et les reins et le ventre se mouvant délicieusement dans la fraîcheur, au milieu de laquelle de petits poissons, des algues... » (MS, 143-144), avant que ses compagnes de marronnage ne l'enduisent « d'une huile balsamique de Judée » dans un rituel digne d'un « mystère » (MS, 144), comme si l'enfant qu'elle porte en elle était promu à une destinée messianique.

56. Extrait du résumé, par André Schwarz-Bart, de son roman en vue de l'adaptation cinématographique (par Claude Faraldo et André Schwarz-Bart). BnF, fonds Schwarz-Bart.

57. Notes autographes (sur une feuille volante arrachée d'un cahier à spirales). BnF, fonds Schwarz-Bart.

58. Extrait de pages autographes sur Solitude à Pointe-à-Pitre, non datées, non titrées et avec pour seul en-tête le chiffre 5. BnF, fonds Schwarz-Bart. Transcription Catherine Rovera.

59. Brouillons autographes. BnF, fonds Schwarz-Bart. Transcription Catherine Rovera.

60. Entretien figurant dans l'annexe de la thèse de Francine Kaufmann (déjà citée, p. 438-448),

p. 441. Reproduit dans Pardès, n 6, 1987, p. 147-158. 


\section{RÉSUMÉS}

On se propose ici de retracer la genèse du roman d'André Schwarz-Bart La Mulâtresse Solitude à la lumière de nombreux documents d'archives : le manuscrit autographe (et en partie calligraphié) du roman, les brouillons et les notes de travail afférentes, mais aussi la correspondance inédite de l'auteur avec les éditions du Seuil. Cette étude à la fois macro- et micro-génétique permettra de suivre les mutations du projet esthétique d'André Schwarz-Bart - et partant, l'évolution dans sa conception du personnage de Solitude - au fil de la composition de l'œuvre. Nous verrons ainsi que la tentation initiale de réaliser une fresque historique cède progressivement la place à un récit de vie mêlant hagiographie et légende, centré sur une héroïne d'une «infinie vulnérabilité ».

This paper seeks to unveil the long-winded creative process that led to André Schwarz-Bart's 1972 novel La Mulâtresse Solitude. Relying on archival and unpublished materials, namely the autograph manuscript-partly written in calligraphy-and what remains of the author's notes and subsequent drafts, as well as his correspondence with his publishers, the present study documents the shift away from epic historical fiction towards a more intimate approach and the imaginary life story of a strong-willed but reluctant heroine characterized by unfathomable vulnerability.

\section{INDEX}

Mots-clés : Schwarz-Bart André, Mulâtresse Solitude, avant-texte, correspondance, cycle antillais, esclavage, exogénèse, histoire, réalisme magique, révolution, technique narrative

\section{AUTEUR}

\section{CATHERINE ROVERA}

CATHERINE ROVERA est maître de conférences en langue et littérature anglaise à l'Université ParisDauphine et responsable de l'équipe « Joyce et le Modernisme Anglophone » à l'Institut des textes et manuscrits modernes. Elle est notamment l'auteur d'une monographie, Genèses d'une folie créole: Jean Rhys et Jane Eyre (Hermann, 2015), et a coédité un volume de critique génétique, Genesis and Revision in Modern British and Irish Writers (Palgrave Macmillan, 2020). Elle s'intéresse au fonds Simone et André Schwarz-Bart et plus spécifiquement à la genèse du cycle antillais. 